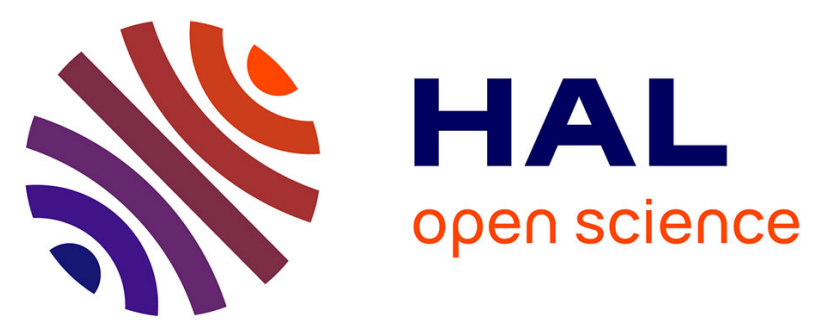

\title{
Activin type IIB receptor blockade does not limit adenosine triphosphate supply in mouse skeletal muscle in Vivo
}

Nelly Béchir, Émilie Pecchi, Christophe Vilmen, Monique Bernard, David Bendahan, Benoit Giannesini

\section{To cite this version:}

Nelly Béchir, Émilie Pecchi, Christophe Vilmen, Monique Bernard, David Bendahan, et al.. Activin type IIB receptor blockade does not limit adenosine triphosphate supply in mouse skeletal muscle in Vivo. Muscle \& nerve. Supplement., 2018, 58 (6), pp.834-842. 10.1002/mus.26306 . hal-03548285

\section{HAL Id: hal-03548285 https://hal.science/hal-03548285}

Submitted on 30 Jan 2022

HAL is a multi-disciplinary open access archive for the deposit and dissemination of scientific research documents, whether they are published or not. The documents may come from teaching and research institutions in France or abroad, or from public or private research centers.
L'archive ouverte pluridisciplinaire $\mathbf{H A L}$, est destinée au dépôt et à la diffusion de documents scientifiques de niveau recherche, publiés ou non, émanant des établissements d'enseignement et de recherche français ou étrangers, des laboratoires publics ou privés. 


\title{
ACTIVIN TYPE IIB RECEPTOR BLOCKADE DOES NOT LIMIT ADENOSINE TRIPHOSPHATE SUPPLY IN MOUSE SKELETAL MUSCLE IN VIVO
}

\author{
NELLY BÉCHIR, MSc, ÉMILIE PECCHI, PhD, CHRISTOPHE VILMEN, MSc, MONIQUE BERNARD, PhD, \\ DAVID BENDAHAN, PhD, and BENOÎT GIANNESINI, PhD \\ Aix-Marseille Univ, CNRS, CRMBM, Marseille, France \\ Accepted 15 July 2018
}

\begin{abstract}
Introduction: Postnatal activin/myostatin type IIB receptor (ActRIIB) blockade increases skeletal muscle mass and strength but also increases muscle fatigability and impairs oxidative metabolism. The objective of this study was to determine in vivo whether this increased fatigability is due to energy supply limitation. Methods: The impact of 8-week ActRIIB blockade with soluble receptor (sActRIIB-Fc) on muscle function and adenosine triphosphate (ATP) fluxes was investigated noninvasively by using multimodal magnetic resonance and indirect calorimetry measurements in wild-type mice. Results: Activin/myostatin type IIB receptor blockade reduced $(-41 \%)$ the muscle apparent mitochondrial capacity and increased $(+11 \%)$ the basal body energy expenditure. During a fatiguing exercise, ActRIIB blockade decreased both oxidative ATP production rate $(-32 \%)$ and fatigue resistance $(-36 \%)$, but these changes affected neither the total ATP production rate nor the contractile ATP cost. Discussion: These findings demonstrate that the increased fatigability after ActRIIB blockade is not due to limitation in energy supply and/or disturbance in contractile ATP cost.
\end{abstract}

Muscle Nerve 58:834-842, 2018

Skeletal muscle overgrowth is limited by various mechanisms, especially through the activin type IIB receptor (ActRIIB), a transmembrane serine-threonine kinase receptor highly expressed in skeletal muscle and adipose tissue. Activin type IIB receptor mediates the signaling for secreted molecules of the transforming growth factor- $\beta$ family involved in the negative regulation of muscle development, the most important being myostatin (GDF-8). ${ }^{1}$ Postnatal blockade of ActRIIB signaling by using systemic injection of soluble ActRIIB decoy receptor (sActRIIB-Fc) or neutralizing antibodies against ActRIIB has been shown to induce a rapid and massive muscle hypertrophy leading to absolute strength improvement in healthy human and in normal and dystrophic mice..$^{2-9}$ These findings have raised the possibility of pharmaceutical

Additional supporting information may be found in the online version of this article.

Key words: activin myostatin receptor, energy expenditure, exercise, fatigue, muscle wasting therapy, oxidative capacity

Abbreviations: ${ }^{31} \mathrm{P}, 31$-phosphorus; ActRIIB, activin type IIB receptor; ANCOVA, analysis of covariance; ATP, adenosine triphosphate; EE, energy expenditure; FID, free induction decays; MR, magnetic resonance; PBS, phosphate-buffered saline; $\mathrm{PCr}$, phosphocreatine; $\mathrm{Pi}$, inorganic phosphate; sActRIIB-Fc, soluble ActRIIB decoy receptor; SR, sarcoplasmic reticulum; $\mathrm{VCO}_{2}, \mathrm{CO}_{2}$ production; $\mathrm{VO}_{2}$, oxygen consumption

Funding: This work was financially supported by the French Muscular Dystrophy Association (AFM-Téléthon; to BG) and the France Life Imaging network (Grant ANR-11-INBS-0006; to MB).

Conflicts of Interest: None of the authors have any conflicts of interest to disclose.

Correspondence to: B. Giannesini; e-mail: benoit.giannesini@univ-amu.fr

(C) 2018 Wiley Periodicals, Inc.

Published online 19 July 2018 in Wiley Online Library (wileyonlinelibrary.

com). DOI 10.1002/mus.26306 blockade of ActRIIB as a promising means for improving the quality of life and reducing mortality of patients with muscle wasting associated with conditions such as neuromuscular disorders, cancer, human immunodeficiency virus, and sarcopenia. ${ }^{4,10-12}$

The beneficial effect of ActRIIB blockade on muscle strength is limited by concomitant impairments in mechanical performance and bioenergetics. Activin type IIB receptor inhibition in healthy mice has been shown to reduce fatigue resistance in hind limb muscle electrically stimulated in situ ${ }^{2,9}$ and during running tests. ${ }^{3}$ Nevertheless, the origin of this increased fatigability remains unknown. Long-term sActRIIB-Fc treatment in mice has been reported to reduce the expression of genes involved in oxidative phosphorylation $^{13,14}$ (via reduction of proliferator-activated receptors and peroxisome-proliferator-activated receptor (PPAR)- $\gamma$-coactivator $1 \alpha$ as a consequence of PPAR- $\beta$ downregulation), the level of porin $^{3}$ (a mitochondrial protein involved in adenosine triphosphate [ATP] transport between the mitochondrial matrix and the cytosol), and mitochondrial oxidative capacity in vivo, ${ }^{9}$ hence indicating mitochondrial dysfunction. Because limitation in energy supply during muscular activity is considered a major contributor to the development of fatigue,${ }^{15,16}$ this raises the question regarding whether a link exists between increased fatigability and mitochondrial dysfunction in muscle undergoing ActRIIB blockade. This warrants consideration not only to facilitate a better understanding of the physiological impact of ActRIIB blockade but also to clarify whether the upregulation of oxidative metabolism would be justified in that context. Such an approach has been envisaged as complementary to ActRIIB blockade to reverse mitochondrial dysfunction; ${ }^{17}$ however, no study has yet been devoted to the investigation of muscle performance with respect to energy fluxes; the effect of the impact of mitochondrial dysfunction induced by ActRIIB blockade over energy expenditure in vivo has not been well studied and remains controversial. Indirect calorimetry experiments have shown that 4-week ActRIIB blockade does not change basal oxygen consumption per animal despite substantial muscle hypertrophy and body weight increase, thereby suggesting that muscle mitochondrial dysfunction does not affect global metabolic rate. ${ }^{18}$ On the contrary, it has been proposed on the basis of similar experiments that ActRIIB blockade reduces energy 
efficiency, ${ }^{19}$ which echoes previous studies in myostatin-deficient mice showing that muscle hypertrophy is associated with a dramatic increase in ATP cost of contraction. ${ }^{20,21}$ Moreover, because energy cost of contraction is an important factor in muscle performance and physical capacity, ${ }^{22-24}$ any increase in this variable might contribute to the increased fatigability induced by ActRIIB blockade.

In this study, we noninvasively investigated the impact of ActRIIB blockade on muscle function and bioenergetics in normal mice. In particular, we sought to determine whether a limitation in energy supply and/or an increase in contractile ATP cost could explain the increase in muscle fatigability already reported in this model. For this purpose, we have used a global in vivo approach, assessing wholebody energy expenditure and ATP production rates in working muscle.

\section{MATERIALS AND METHODS}

Animal Care and Feeding. Fifteen C57BL/6 3-month-old female mice (Janvier Labs, Le Genest Saint Isle, France) were used for these experiments, which were conducted in strict accordance with the guidelines of the European Communities Council Directive 86/609/EEC for care and use of laboratory animals. All experiments were performed according to protocols reviewed and approved by the institutional animal care committee of Aix-Marseille University (93-21122012) and under the supervision of $B$. Giannesini (license number $13.1642008 / 11 / 25)$. Mice were housed 3 or 4 per cage in an environmentally controlled facility (12-h light/dark cycle, $22^{\circ} \mathrm{C}$ ) with free access to commercial standard food and water. After experiments, animals were humanely killed by cervical dislocation after isoflurane anesthesia, and gastrocnemius muscles were immediately removed, freeze clamped with nitrogen-chilled metal tongs, and stored at $-80^{\circ} \mathrm{C}$ for in vitro measurement.

ActRIIB Signaling Blockade. The blockade was performed by using a soluble fusion protein generated by linking the ActRIIB extracellular domain to the mouse immunoglobulin Fc region (sActRIIB-Fc; provided by Acceleron Pharma, Cambridge, Massachusetts). Mice were randomly assigned to 2 groups and were injected subcutaneously twice weekly for a total of 8 weeks with sActRIIB-Fc $(10 \mathrm{mg} / \mathrm{kg}$ body weight; $n=8)$ or an equal volume of vehicle $(10 \mathrm{mM}$ phosphatebuffered saline [PBS]; control group; $n=7$ ).

\section{NONINVASIVE INVESTIGATION OF GASTROCNEMIUS MUSCLE FUNCTION AND BIOENERGETICS}

Muscle anatomy and changes in bioenergetics associated with force output were investigated with multimodal magnetic resonance (MR) data acquisition throughout a standardized fatiguing bout of exercise consisting of $6 \mathrm{~min}$ of repeated maximal isometric contractions induced by transcutaneous electrostimulation at a frequency of $1.7 \mathrm{~Hz}$.

Each mouse was initially anesthetized in an induction chamber by using an air flow $(3 \mathrm{~L} / \mathrm{min})$ containing $4 \%$ isoflurane. The left hind limb was shaved, and electrode cream was applied at the knee and heel regions to optimize electrostimulation. The anesthetized animal was then placed supine in a custom-built experimental cradle that had been designed for the noninvasive MR investigation of gastrocnemius muscle function. ${ }^{25}$ Corneas were protected from drying by applying ophthalmic cream, and the animal's head was placed in a facemask that was continuously supplied with $1.75 \%$ isoflurane in $33 \% \mathrm{O}_{2}(0.2 \mathrm{~L} / \mathrm{min})$ and $66 \% \mathrm{~N}_{2} \mathrm{O}(0.4 \mathrm{~L} / \mathrm{min})$. The left foot was positioned on the pedal of an ergometer, the lower hind limb was centered inside a 20-mmdiameter ${ }^{1} \mathrm{H}$ Helmholtz imaging coil, and the belly of the gastrocnemius muscle was located above an elliptic $\left(8 \times 12 \mathrm{~mm}^{2}\right)$ 31-phosphorus $\left({ }^{31} \mathrm{P}\right)$-MR spectroscopy surface coil. Body temperature was monitored and maintained at physiological level throughout the entire experiment by using a feedback loop including electrical heating, a temperature control unit, and a rectal thermometer.

Muscle contractions were achieved in vivo by using 2 rod-shaped transcutaneous electrodes integrated in the experimental cradle and connected to a constantcurrent stimulator (DS7A; Digitimer, Herthfordshire, United Kingdom): 1 electrode was located above the knee and the other electrode was placed under the heel. Mechanical response to electrostimulation was measured by amplifying (operational amplifier AD620; Analog Devices, Norwood, Massachusetts) the electrical signal coming out from the force transducer. The signal was then digitized (PCI-6220; National Instrument, Austin, Texas) and continuously monitored and recorded on a personal computer in WinATS version 6.5 (Sysma, Aix-en-Provence, France). The digitalized signal was converted to force according to a linear calibration curve. At the beginning of each experiment, resting muscle was passively stretched by adjusting the angle between the foot and the lower hind limb to reach maximal isometric twitch amplitude after supramaximal square wave pulses (1-ms duration). The individual maximal electrostimulation intensity was determined by progressively increasing the stimulus intensity until there was no further peak twitch force increase.

Magnetic resonance explorations were performed in the 4.7-Tesla horizontal magnet of a 47/30 Biospec Avance MR system (Bruker, Karlsruhe, Germany) equipped with a Bruker 120-mm BGA12SL (200 mT/m) gradient insert. Ten consecutive noncontiguous axial slices (1-mm thickness, 0.5-mm interslice gap) covering the region from the knee to the ankle were selected across the hind limb. Magnetic resonance images of these slices (rapid acquisition with relaxation enhancement sequence; 8 echoes; 67.9 -ms effective echo time; 16.7-ms actual echo time; 2,000-ms repetition time; 1 accumulation; $20 \times 15 \mathrm{~mm}^{2}$ field of view; $256 \times 256$ matrix size) were recorded at rest. 31-phosphorus-MR spectra 
(8 kHz sweep width; 2,048 data points) from the gastrocnemius region were dynamically acquired at rest (6 min), during the 6-min fatiguing bout of exercise, and during the following 15 -min recovery period. Magnetic resonance data acquisition was gated to muscle electrostimulation for reducing potential motion artifacts due to contraction. A fully relaxed spectrum (12 scans, 20-s repetition time) was acquired at rest, followed by a total of 768 saturated free induction decays (FID; 1.875 -s repetition time). The first 64 FIDs were acquired at rest and added together. The next 192 FIDs were acquired during the stimulation period and were added by packets of 32 , allowing a temporal resolution of $60 \mathrm{~s}$. The remaining 512 FIDs were obtained during the poststimulation recovery period and were added as 7 packets of 32 FIDs followed by 3 packets of 64 FIDs (120-s temporal resolution) and 1 packet of 96 FIDs (180-s temporal resolution).

Magnetic resonance data were processed by using custom-written analysis algorithms developed under the IDL environment (Interactive Data Language; Harris Geospacial Solutions, Broomfield, Colorado). For each MR image, the region of interest was manually outlined so that the corresponding crosssectional area of the gastrocnemius muscle was measured. Muscle volume was then calculated by adding the volumes included between consecutive slices by using the truncated cone formula. Intracellular $\mathrm{pH}$ was calculated from the chemical shift of the inorganic phosphate $\left(\mathrm{P}_{\mathrm{i}}\right)$ signal relative to phosphocreatine (PCr). ${ }^{26}$ Relative concentrations of $\mathrm{PCr}, \mathrm{P}_{\mathrm{i}}$, and ATP were obtained from ${ }^{31} \mathrm{P}-\mathrm{MR}$ spectra by a timedomain fitting routine by using the AMARES-MRUI Fortran code and appropriate prior knowledge for the ATP multiplets. ${ }^{27}$ Absolute amounts of phosphorylated compounds were expressed relative to a resting $\beta$-ATP concentration determined in muscle extract by using a bioluminescence-based method (Supp. Info.). The rates of ATP production from oxidative and anaerobic pathways were calculated in vivo during the fatiguing bout of exercise according to the quantitative interpretation of bioenergetics data $^{28,29}$ (Supp. Info). Adenosine triphosphate cost of contraction was calculated as total ATP production (oxidative plus anaerobic) scaled to force generation during the same period of time.

In Vivo Quantitation of Body Fat Content. Magnetic resonance image acquisition was performed with a whole-body imaging coil (PRK 200 RES 200; Bruker) inserted in the 4.7-Tesla horizontal magnet of the 47/30 Biospec Avance MR system. Mice were kept anesthetized by using isoflurane inhalation as previously described. Axial MR images were acquired across the whole body (excluding the tail) by using a high-resolution 3-dimensional (turbo spin echo) sequence (5.53-ms echo time; 77.85-ms effective echo time; 300-ms repetition time; 2 averages; $40 \times 40 \times 80 \mathrm{~mm}^{3}$ field of view; $128 \times 128 \times 64$ matrix size). Fat volume was quantitated by using an automatic segmentation method that was based on a pixel-intensity analysis of MR images with the FMRIB Software Library v5.0.2.2, (Oxford University, United Kingdom; http://www.fmrib.ox.ac.uk/fsl). Fat mass was calculated by using a density of $0.92 \mathrm{~g} / \mathrm{cm}^{3}$ for adipose tissue. ${ }^{30}$ Adiposity index was calculated as fat mass relative to total body weight.

Body Energy Expenditure. Measurements were performed at room temperature $\left(23^{\circ} \mathrm{C}\right)$ by using a 2-chamber Oxylet system (Panlab, Barcelona, Spain) with a constant inlet flow of room air $(5 \mathrm{ml} / \mathrm{min})$. Animals were individually acclimated and kept for $24 \mathrm{~h}$ in metabolic cages with free access to food and water. Oxygen consumption $\left(\mathrm{VO}_{2}\right)$ and $\mathrm{CO}_{2}$ production $\left(\mathrm{VCO}_{2}\right)$ were continuously analyzed by using the following sequence: $5 \mathrm{~min}$ from cage No. 1, $5 \mathrm{~min}$ from cage No. 2, and $5 \mathrm{~min}$ from room air. Energy expenditure (EE) was calculated as EE = $(3.815+1.232 \mathrm{RQ}) \times \mathrm{VO}_{2}$, in which $\mathrm{RQ}$ is the respiratory quotient of $\mathrm{VCO}_{2}$ to $\mathrm{VO}_{2}$. Ambulatory activity (locomotion and rearing) was determined simultaneously with the collection of indirect calorimetry data.

Statistical Analysis. All data are mean \pm SEM (except in Fig. 1A, in which data are reported as mean \pm SD for clarity). The overall time courses of variables changing with respect to time were compared with two-factor (group $\times$ time) analysis of variance with repeated measures on time followed when appropriated $(P$-value $<0.05)$ by Tukey-Kramer post hoc multiple comparison tests for determining pairwise differences between groups. The influence of body mass or lean mass on energy expenditure was performed by using analysis of covariance (ANCOVA). ${ }^{31,32}$ Other variables were compared with paired or unpaired two-tailed Student's $t$ tests. The significance level was set at $P<0.05$.

\section{RESULTS}

Anatomical Changes And Body Energy Expenditure.

Mice treated with sActRIIB-Fc had a significant increase in body weight that began after a week of treatment (Fig. 1A). At the termination of the 8-week treatment period, body weight (Fig. 1A) and body lean mass (Fig. 1B) were larger $(+32 \%$ and $+33 \%$, respectively) in animals treated with sActRIIBFc, whereas the body fat content did not differ between groups (Fig. 1C). As a result, the adiposity index was $14 \%$ lower in mice treated with sActRIIBFc (Fig. 1D). Treatment with sActRIIB-Fc also dramatically increased $(+60 \%)$ the gastrocnemius 

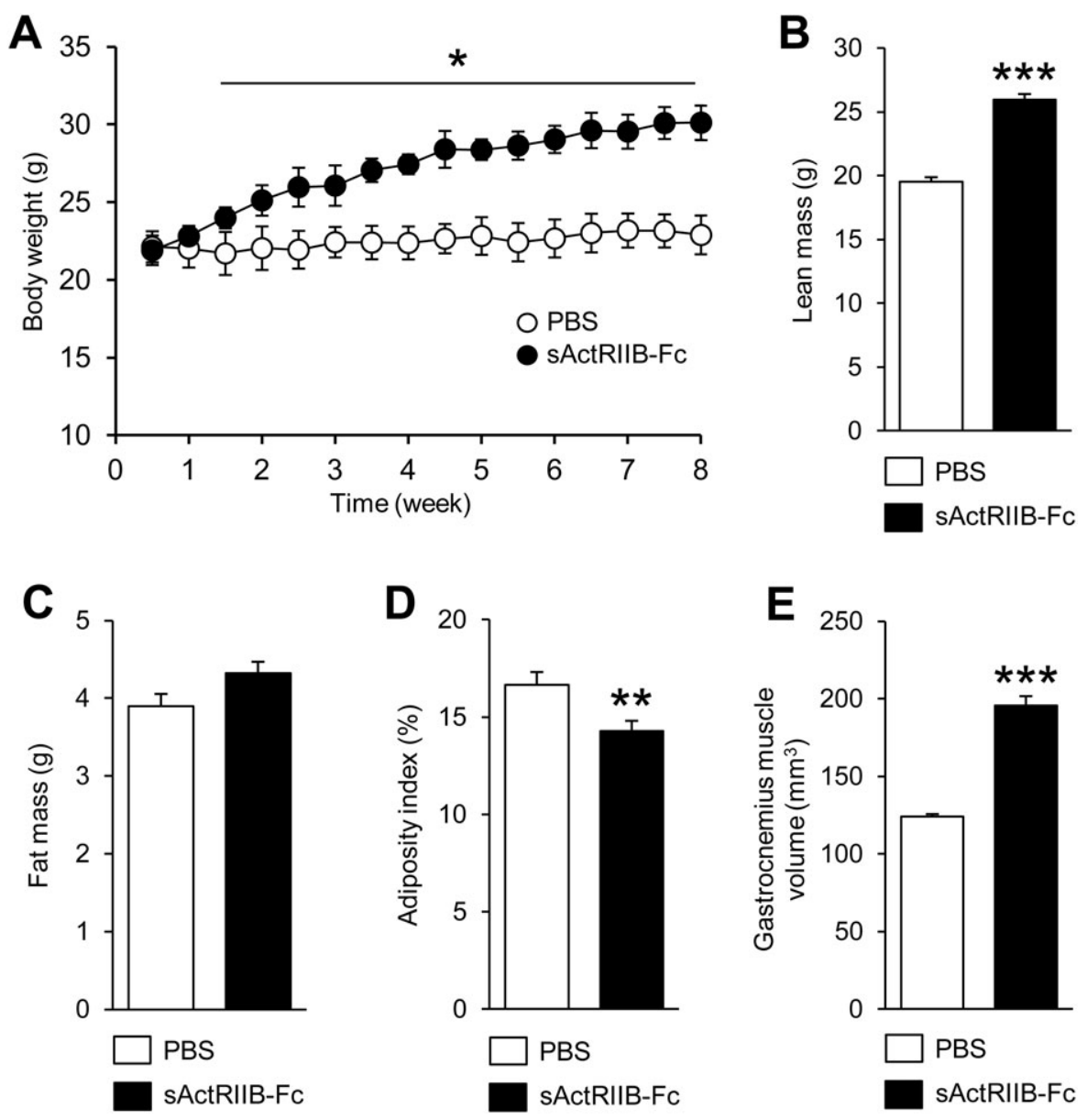

FIGURE 1. Physiological changes. Changes in body weight throughout the 8-week treatment with PBS or sActRIIB-Fc (A). Lean mass (B), fat mass (C), adiposity index (D), and gastrocnemius muscle volume (E) at the end of the 8-week treatment. All data are mean \pm SEM (except for $\mathbf{A}$ in which data are reported as mean \pm SD for clarity). $* P<0.05, * * P<0.01, * * * P<0.001$ vs. PBS-treated animals. PBS, phosphate-buffered saline; sActRIIB-Fc, soluble activin type IIB decoy receptor.

muscle volume (Fig. 1E). $\mathrm{O}_{2}$ consumption (100 $\pm 4 \mathrm{ml} / \mathrm{h}, \quad \mathrm{PBS} ; \quad 111 \pm 3 \mathrm{ml} / \mathrm{h}, \quad$ sActRIIB-Fc),$\quad \mathrm{CO}_{2}$ release $(78 \pm 3 \mathrm{~mL} / \mathrm{h}, \mathrm{PBS} ; 88 \pm 3 \mathrm{ml} / \mathrm{h}$ sActRIIB-Fc), and energy expenditure $(0.48 \pm 0.02 \mathrm{kcal} / \mathrm{h}, \mathrm{PBS}$;
$0.53 \pm 0.02 \mathrm{kcal} / \mathrm{h}$, sActRIIB-Fc) per animal were larger $(+11 \%,+13 \%$ and $+11 \%$, respectively) in the group receiving sActRIIB-Fc. After adjustment for total body weight by ANCOVA (Fig. 2A), mice treated with
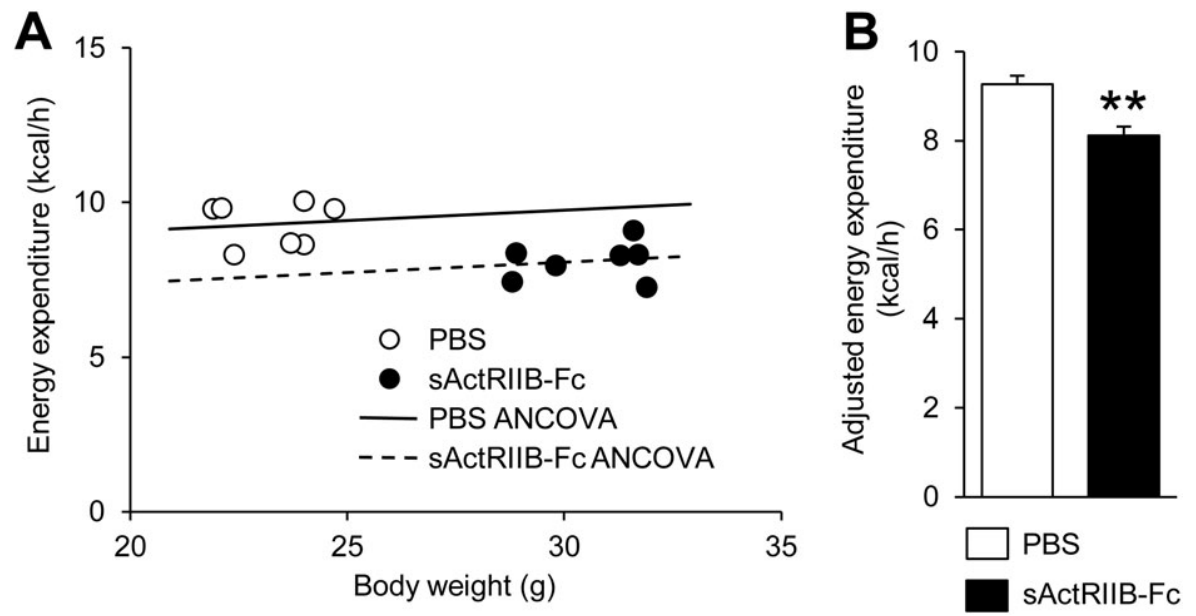

FIGURE 2. Adjustment of energy expenditure for body weight. Energy expenditure per animal during a 24-h period was plotted against body weight (A), and group comparison was adjusted for body weight by using ANCOVA (B). Data are mean \pm SEM. $* * P<0.01$ vs. PBS-treated animals. ANCOVA, analysis of covariance; PBS, phosphate-buffered saline; sActRIIB-Fc, soluble activin type IIB decoy receptor. 

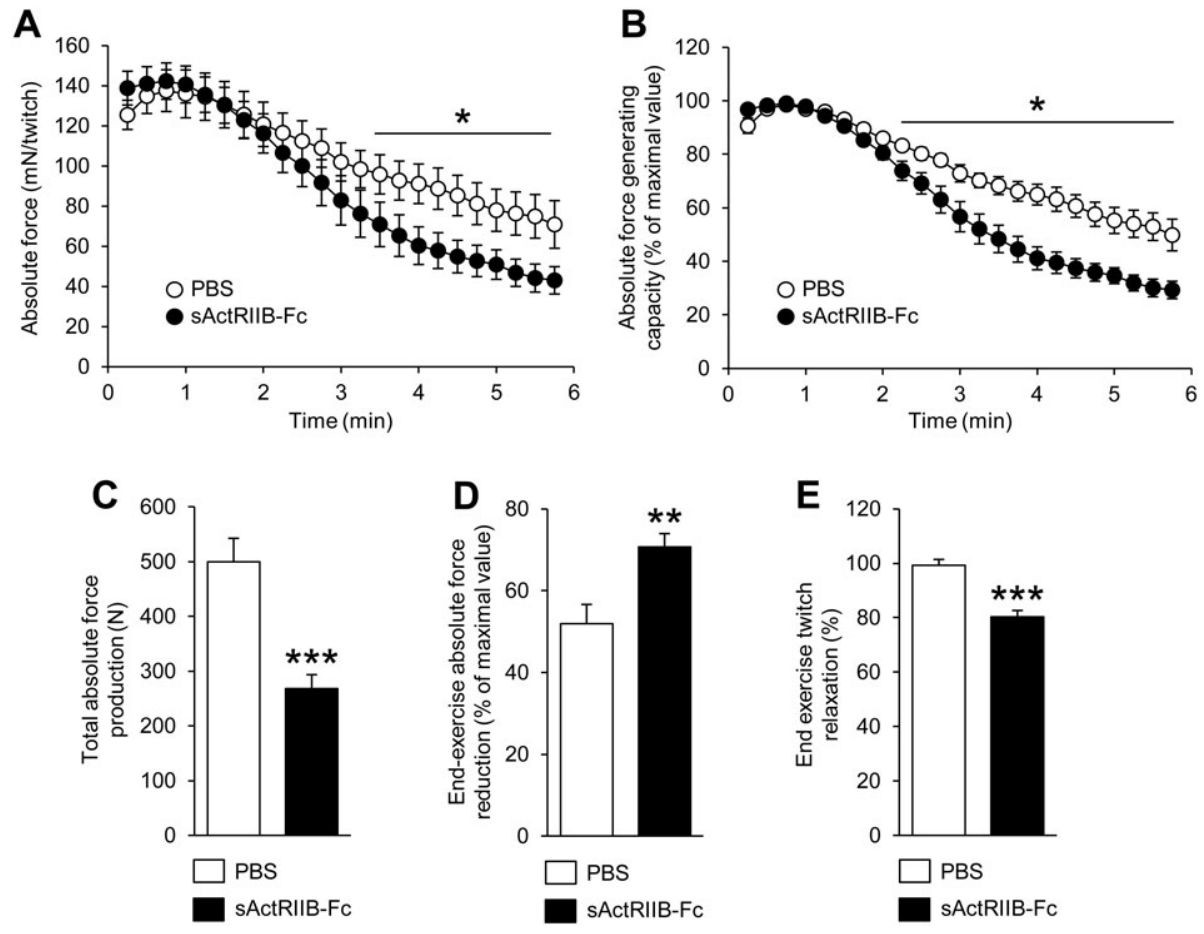

FIGURE 3. In vivo gastrocnemius muscle force-generating capacity. Absolute force (A) and absolute force-generating capacity (B) throughout the 6-min fatiguing bout of exercise performed in vivo simultaneously with ${ }^{31} \mathrm{P}-\mathrm{MR}$ spectra acquisition. Total amount of absolute force produced during the whole exercise (C) and extent of force reduction (D) and muscle relaxation (E) measured at the end of exercise. Data are mean \pm SEM. $* P<0.05$, $* * P<0.01$, $* * * P<0.001$ vs. PBS-treated animals. ${ }^{31} \mathrm{P}-\mathrm{MR}$, 31-phosphorus; MR, magnetic resonance; PBS, phosphate-buffered saline; sActRIIB-Fc, soluble activin type IIB decoy receptor.

sActRIIB-Fc exhibited lower energy expenditure (Fig. 2B). Adjustment for lean mass produced similar differences $(9.3 \pm 0.2 \mathrm{kcal} / \mathrm{h}, \mathrm{PBS} ; 8.1 \pm 0.2 \mathrm{kcal} / \mathrm{h}$, sActRIIB-Fc). There were no differences between groups for respiratory quotient $(0.78 \pm 0.01$, PBS; $0.80 \pm 0.02$, sActRIIB-Fc), horizontal activity (581 \pm 92 arbitrary unit, PBS; $806 \pm 190$ arbitrary unit, sActRIIBFc), and rearing (110 \pm 19 counts/h, PBS; $92 \pm 15$ counts/h, sActRIIB-Fc).

\section{Gastrocnemius Muscle Function And Bioenergetics.}

Treatment with sActRIIB-Fc increased by $22 \%$ the gastrocnemius muscle tension $(122 \pm 7 \mathrm{mN}, \mathrm{PBS} ; 150 \pm 7$ $\mathrm{mN}$, sActRIIB-Fc) produced during a single twitch performed before MR multimodal acquisition but did not affect the corresponding contraction $(47 \pm 2 \mathrm{~ms}$, PBS; $50 \pm 2 \mathrm{~ms}$, sActRIIB-Fc) and half-relaxation (30 \pm 2 ms, PBS; $35 \pm 2$ ms, sActRIIB-Fc) times. Changes in absolute force and force-generating capacity throughout the 6-min fatiguing bout of exercise are illustrated in Figure 3A,B. In mice treated with sActRIIB-Fc, total force produced during the whole exercise was $46 \%$ lower compared with the control group (Fig. 3C). Furthermore, the extent of force reduction at the end of exercise was $36 \%$ larger in sActRIIB-Fc-treated mice (Fig. 3D), indicating a lower resistance to fatigue. Twitch relaxation (i.e., the return to rest tension between consecutive twitches) was achieved in the early stage of exercise for both groups. At the end of exercise, relaxation reached $99 \% \pm 2 \%$ and $80 \% \pm 2$ $\%$ in PBS- and sActRIIB-Fc-treated mice, respectively (Fig. 3E).

Mitochondrial oxidative capacity and metabolic fluxes were inferred from the in vivo changes in $\mathrm{PCr}$
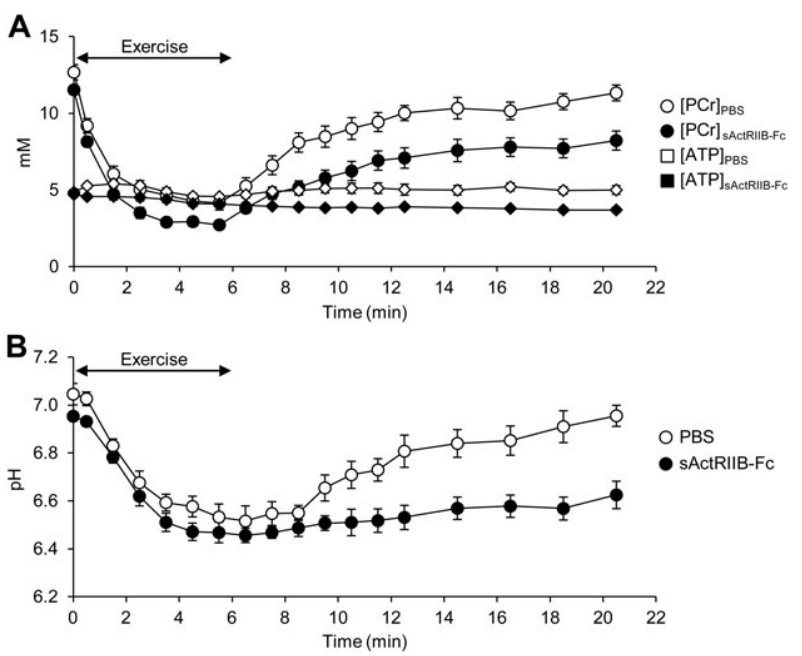

FIGURE 4. Noninvasive investigation of gastrocnemius muscle bioenergetics with dynamic ${ }^{31} \mathrm{P}-\mathrm{MR}$ spectroscopy. Changes in PCr and ATP levels (A) and $\mathrm{pH}$ (B) were measured throughout the 6-min fatiguing bout of exercise and the 15-min postexercise recovery period. For each panel, the first time-point $(t=0)$ indicates the basal value. Data are mean \pm SEM. ATP, adenosine triphosphate; ${ }^{31} \mathrm{P}-\mathrm{MR}, 31$-phosphorus magnetic resonance; PBS, phosphate-buffered saline; PCr, phosphocreatine; sActRIIB-Fc, soluble activin type IIB decoy receptor 

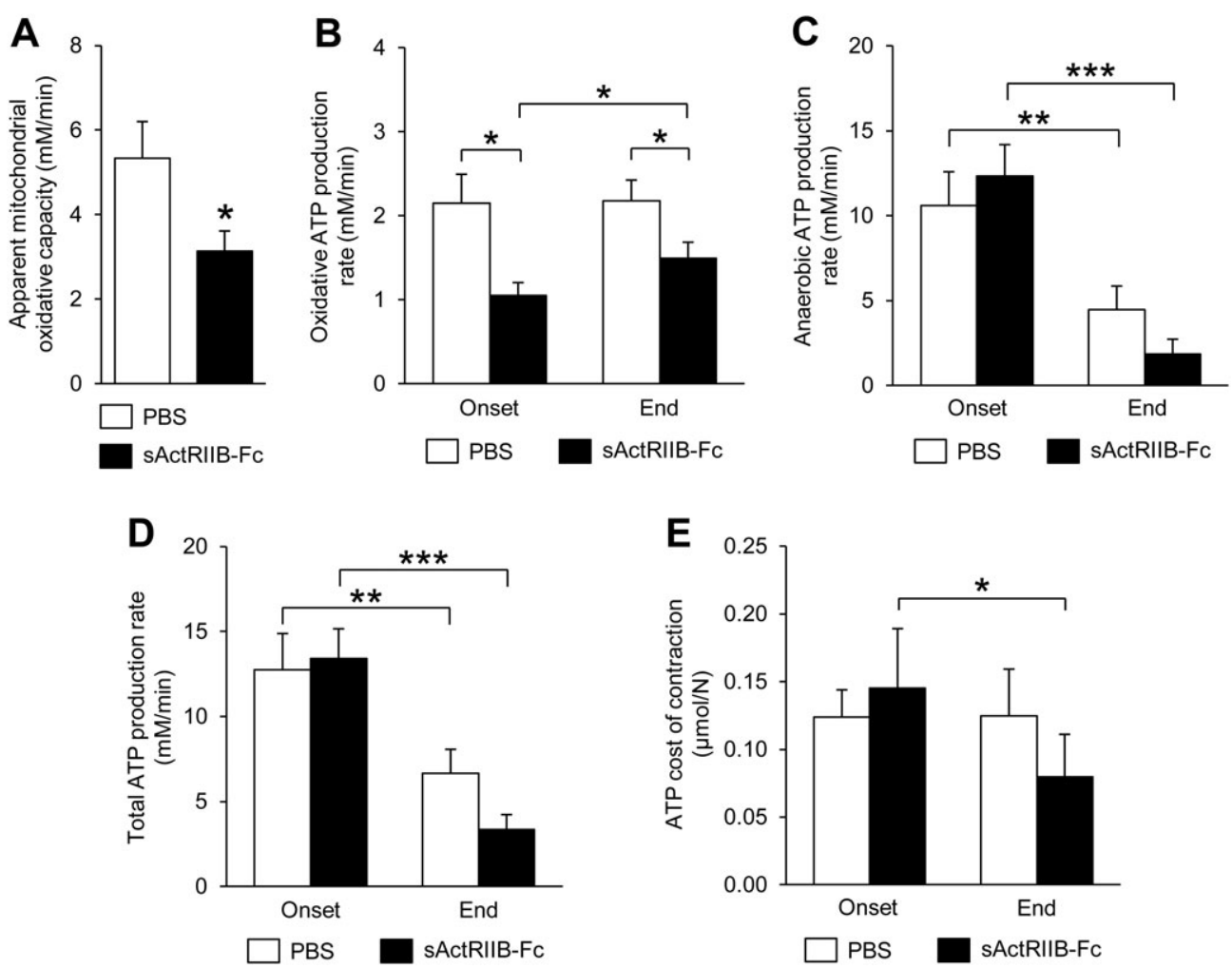

FIGURE 5. Metabolic fluxes and ATP cost of contraction. Apparent mitochondrial oxidative capacity (A), oxidative (B) and anaerobic (C) ATP production rates, total ATP production rate (D), and ATP cost of contraction (E) calculated at the start and at the end of the 6-min fatiguing bout of exercise. Data are mean \pm SEM. $* P<0.05, * * P<0.01, * * * P<0.001$ vs. PBS-treated animals. ATP, adenosine triphosphate; PBS, phosphate-buffered saline.

and ATP levels (Fig. 4A) and pH (Fig. 4B) throughout the rest, exercise, and postexercise recovery periods. The apparent mitochondrial oxidative capacity was $41 \%$ lower in mice treated with sActRIIB-Fc (Fig. 5A) and was associated with a reduction of the oxidative ATP production rate at the start $(-51 \%)$ and at the end $(-32 \%)$ of the exercise (Fig. 5B). In contrast, sActRIIB-Fc affected neither the anaerobic ATP production rate (Fig. 5C) nor the total ATP production rate (Fig. 5D) at the start and end of exercise. Overall, there were no differences between groups for the ATP cost of contraction at the start and at the end of the exercise (Fig. 5E). However, the ATP cost of contraction was decreased by $44 \%$ between the start and the end of the exercise in sActRIIB-Fc-treated mice, whereas it remained unchanged during this interval in PBS-treated mice (Fig. 5E).

\section{DISCUSSION}

The major findings from this study are that longterm ActRIIB blockade reduces the muscle apparent mitochondrial capacity in vivo and disturbs the basal body energy expenditure, whereas the voluntary locomotor activity remains unchanged. In exercising muscle, ActRIIB blockade enhances the development of fatigue and reduces the oxidative ATP production rate, but this reduction affects neither energy supply nor the ATP cost of contraction. An additional important finding is that ActRIIB blockade impairs twitch relaxation in fatigued muscle.

In agreement with previously published reports, we found that ActRIIB blockade increases muscle strength and produces dramatic morphological changes, including muscle hypertrophy and increase in both lean mass and body weight, whereas body fat content remains unchanged. ${ }^{18,19,33,34}$ Just as we had expected, we found that the blockade strongly reduces the muscle apparent mitochondrial capacity in vivo, thereby corroborating previous in vitro studies in mice showing that biomarkers of oxidative function are impaired after postnatal ActRIIB inhibition $^{3,13,14}$ and myostatin gene deficiency. ${ }^{21,35,36}$ Because skeletal muscle plays an important role in body energy homeostasis, our results suggest that long-term sActRIIB-Fc treatment affects basal energy expenditure, similarly to what has been reported in myostatin-null mice. ${ }^{37,38}$ We performed indirect calorimetry experiments to test this assumption and found that ActRIIB blockade increases basal oxygen consumption and energy expenditure per animal. Our findings differ from previous experiments showing that ActRIIB blockade has no effect upon basal $\mathrm{VO}_{2}$ per mouse. ${ }^{18}$ However, this discrepancy could be linked to the duration of treatment (i.e., 8 weeks here vs. 4 weeks in the previous experiments) $i^{18}$ our results are closer to those obtained in myostatin- 
deficient mice in which basal metabolic rate is increased relative to wild-type controls. ${ }^{38}$ We found that this increase $(11 \%)$ in energy expenditure was not proportional to the rise $(32 \%-33 \%)$ in both body weight and lean mass. It is tempting to attribute this imbalance to mitochondrial dysfunction, but this phenomenon might also result from the differences in oxygen consumption among tissues. Basal oxygen consumption in skeletal muscle is much lower than the corresponding consumption in high-energy tissues such as brown adipose tissue and heart so that muscle hypertrophy is not expected to induce a substantial rise in resting energy consumption. Thus, after adjustment for body weight or lean mass by covariance analysis, we found that basal energy expenditure was reduced in animals treated with sActRIIB-Fc. This reduction occurred, however, without a corresponding change in voluntary ambulatory activity, which raises the possibility that ActRIIB blockade increases the animal's physical capacity through a reduction in the energy cost of ambulation. These considerations are in opposition to the reduction in muscle fatigue resistance evidenced here and in previous studies in mice treated with ActRIIB blocker. ${ }^{2,3}$ Nevertheless, the situation could be different in exercising muscle because of the large increase in energy demand.

To answer this question, we assessed ATP fluxes at the start (i.e., before fatigue development) and at the end of a fatiguing bout of exercise generating large changes in force production and energy demand. As an illustration, force reduction at the end of exercise reached $52 \%$ and $71 \%$ in PBS and sActRIIB-Fc groups, respectively. In mice treated with sActRIIB-Fc, we found that the oxidative ATP production rate was much lower at these 2 times, thereby providing evidence that the deleterious effect of ActRIIB blockade on mitochondrial function directly impacts bioenergetics in working muscle. However, this lower oxidative rate was compensated for by ATP production from anaerobic processes so that total energy production throughout the exercise was not disturbed in animals treated with sActRIIB-Fc. In addition, we found that ActRIIB blockade did not affect the ATP cost of contraction before and after the development of fatigue. Overall, our findings demonstrate that, despite impairing muscle oxidative function, ActRIIB blockade does not limit energy supply during a sustained exercise. However, the mouse gastrocnemius muscle is predominantly glycolytic, so the results of the present study might not be applicable to pure oxidative muscle. In any case, our results support the concept of upregulating oxidative metabolism as a complementary approach to ActRIIB blockade. ${ }^{17}$

We found that the contractile ATP cost decreased by $45 \%$ between the start and the end of the fatiguing exercise in mice treated with sActRIIB-Fc, whereas it did not differ significantly during this interval in control mice. Shift in the pattern of fiber type recruitment and alteration of activationrelaxation processes have been proposed to explain the reduction of contractile ATP cost throughout a fatiguing exercise. ${ }^{39-41}$ Mouse gastrocnemius muscle is a mixed muscle composed mainly of fast glycolytic type-2b fibers (about $85 \%$ ) with low oxidative capacity; the remaining muscle is composed of fastoxidative type-2a fibers with higher oxidative capacity. ${ }^{9,42}$ Because a fiber is more fatigue resistant and contracts more economically when its metabolism is oxidative, ${ }^{43,44}$ change in muscle typology and/or preferential recruitment of type-2a fibers could explain the decrease in contractile ATP cost in fatigued muscle. This was unlikely in the present study for at least 2 reasons. First, we recently demonstrated that an 8-week treatment with sActRIIB-Fc does not affect fiber type distribution in the gastrocnemius muscle of wild-type mice. ${ }^{9}$ Second, our methodology allows stimulation of the whole gastrocnemius muscle, ${ }^{25}$ and there is no reason that 1 type of fiber would be recruited more than the other in sActRIIB-Fc-treated animals compared with controls. On the other hand, we have observed in animals that have been treated with sActRIIB-Fc that twitch relaxation (i.e., the return to rest tension between consecutive twitches) was not fully achieved at the end of exercise, thereby indicating an alteration of calcium fluxes across the sarcoplasmic reticulum (SR) membrane. Such an alteration has been linked to fatigue development and/or structural anomalies at the SR level. ${ }^{45-47}$ It should be noted that the accumulation of tubular aggregates has been observed in the SR calcium release channel of myostatin-deficient mice, and this accumulation could alter calcium handling during excitation-contraction cycles. ${ }^{48,49}$ It is then conceivable that ActRIIB blockade produces similar accumulation of tubular aggregates, hence reducing calcium availability during excitation-contraction cycles. Because $20 \%-50 \%$ of the ATP consumed during muscle contraction is used for calcium pumping across the SR membrane, ${ }^{39,50}$ alteration in the activation-relaxation process might contribute to the reduction in contractile ATP cost in sActRIIB-Fctreated animals.

In conclusion, these data demonstrate in vivo that, despite the decrease in oxidative capacity, muscle fatigue enhancement after ActRIIB treatment cannot be attributed to any limitation in energy supply and/or disturbance in contractile ATP cost. Additional investigations are required for determining the origin of this increased fatigability. This issue warrants consideration even if it can be argued that absolute increase in strength caused by ActRIIB blockade constitutes a functional advantage over the 
increased fatigability. The possibility of attenuating or preventing muscle fatigability is an important clinical consideration for improving the quality of life of patients who are treated for muscle wasting and weakness.

We thank Acceleron Pharma Inc for the gift of sActRIIB-Fc; and Delphine Bastelica and Marie-Christine Alessi are both PhD (NORT, Aix-Marseille Université, INSERM U1062/INRA 1260, Marseille, France) for sharing the indirect calorimetry system.

Ethical Publication Statement: All authors have read the Journal's position on issues involved in ethical publication and affirm that this report is consistent with those guidelines.

\section{REFERENCES}

1. De Caestecker M. The transforming growth factor-beta superfamily of receptors. Cytokine Growth Factor Rev 2004;15(1):1-11.

2. Chiu CS, Peekhaus N, Weber H, Adamski S, Murray EM, Zhang HZ, et al. Increased muscle force production and bone mineral density in ActRIIB-Fc-treated mature rodents. J Gerontol A Biol Sci Med Sci 2013; 68(10):1181-1192

3. Relizani K, Mouisel E, Giannesini B, Hourde C, Patel K, Morales Gonzalez S, et al. Blockade of ActRIIB signaling triggers muscle fatigability and metabolic myopathy. Mol Ther 2014;22(8):1423-1433.

4. Attie KM, Borgstein NG, Yang Y, Condon CH, Wilson DM, Pearsall AE, et al. A single ascending-dose study of muscle regulator ACE-031 in healthy volunteers. Muscle Nerve 2013;47(3):416-423.

5. Lach-Trifilieff E, Minetti GC, Sheppard K, Ibebunjo C, Feige JN, Hartmann S, et al. An antibody blocking activin type II receptors induces strong skeletal muscle hypertrophy and protects from atrophy. Mol Cell Biol 2014;34(4):606-618.

6. Morrison BM, Lachey JL, Warsing LC, Ting BL, Pullen AE, Underwood KW, et al. A soluble activin type IIB receptor improves function in a mouse model of amyotrophic lateral sclerosis. Exp Neurol 2009;217(2):258-268.

7. Bogdanovich S, Krag TO, Barton ER, Morris LD, Whittemore LA, Ahima RS, et al. Functional improvement of dystrophic muscle by myostatin blockade. Nature 2002;420(6914):418-421.

8. Wang $Q$, McPherron AC. Myostatin inhibition induces muscle fibre hypertrophy prior to satellite cell activation. J Physiol (Lond) 2012;590: 2151-2165.

9. Bechir N, Pecchi E, Relizani K, Vilmen C, Le Fur Y, Bernard M, et al. Mitochondrial impairment induced by postnatal ActRIIB blockade does not alter function and energy status in exercising mouse glycolytic muscle in vivo. Am J Physiol Endocrinol Metab 2016;310(7):E539-E549.

10. LeBrasseur NK, Schelhorn TM, Bernardo BL, Cosgrove PG, Loria PM, Brown TA. Myostatin inhibition enhances the effects of exercise on performance and metabolic outcomes in aged mice. J Gerontol A Biol Sci Med Sci 2009;64(9):940-948.

11. Dumonceaux J, Amthor H. Current advances in the development of therapies for neuromuscular disorders based on myostatin signalling, 3rd International Institute of Myology Workshop, Paris, September 12th, 2008. Neuromuscul Disord 2009;19(11):797-799.

12. O'Connell KE, Guo W, Serra C, Beck M, Wachtman L, Hoggatt A, et al. The effects of an ActRIIb receptor Fc fusion protein ligand trap in juvenile simian immunodeficiency virus-infected rhesus macaques. FASEB J 2014.

13. Rahimov F, King OD, Warsing LC, Powell RE, Emerson CP Jr, Kunkel LM, et al. Gene expression profiling of skeletal muscles treated with a soluble activin type IIB receptor. Physiol Genomics 2011;43(8): 398-407.

14. Zhao B, Li EJ, Wall RJ, Yang J. Coordinated patterns of gene expressions for adult muscle build-up in transgenic mice expressing myostatin propeptide. BMC Genomics 2009;10:305.

15. Allen DG, Lannergren J, Westerblad H. The role of ATP in the regulation of intracellular $\mathrm{Ca}^{2+}$ release in single fibres of mouse skeletal muscle. J Physiol (Lond) 1997;498:587-600.

16. Sahlin K, Tonkonogi M, Soderlund K. Energy supply and muscle fatigue in humans. Acta Physiol Scand 1998;162(3):261-266.

17. Pauly M, Chabi B, Favier FB, Vanterpool F, Matecki S, Fouret G, et al. Combined strategies for maintaining skeletal muscle mass and function in aging: myostatin inactivation and AICAR-associated oxidative metabolism induction. J Gerontol A Biol Sci Med Sci 2015;70(9):1077-1087.

18. Akpan I, Goncalves MD, Dhir R, Yin X, Pistilli EE, Bogdanovich S, et al. The effects of a soluble activin type IIB receptor on obesity and insulin sensitivity. Int J Obes (Lond) 2009;33(11):1265-1273.

19. Fournier B, Murray B, Gutzwiller S, Marcaletti S, Marcellin D, Bergling $\mathrm{S}$, et al. Blockade of the activin receptor IIb activates functional brown adipogenesis and thermogenesis by inducing mitochondrial oxidative metabolism. Mol Cell Biol 2012;32(14):2871-2879.

20. Giannesini B, Vilmen C, Amthor H, Bernard M, Bendahan D. Lack of myostatin impairs mechanical performance and ATP cost of contraction in exercising mouse gastrocnemius muscle in vivo. Am J Physiol Endocrinol Metab 2013;305(1):E33-E40.

21. Mouisel E, Relizani K, Mille-Hamard L, Denis R, Hourde C, Agbulut O, et al. Myostatin is a key mediator between energy metabolism and endurance capacity of skeletal muscle. Am J Physiol Regul Integr Comp Physiol 2014;307(4):R444-R454.

22. Lauretani F, Russo CR, Bandinelli S, Bartali B, Cavazzini C, Di Iorio A, et al. Age-associated changes in skeletal muscles and their effect on mobility: an operational diagnosis of sarcopenia. J Appl Physiol 2003; 95(5):1851-1860.

23. Lauretani F, Russo CR, Bandinelli S, Bartali B, Cavazzini C, Di Iorio A et al. Age-associated changes in skeletal muscles and their effect on mobility: an operational diagnosis of sarcopenia. J Appl Physiol (1985) 2003;95(5):1851-1860.

24. Tevald MA, Foulis SA, Lanza IR, Kent-Braun JA. Lower energy cost of skeletal muscle contractions in older humans. Am J Physiol Regul Integr Comp Physiol 2010;298(3):R729-R739.

25. Giannesini B, Vilmen C, Le Fur Y, Dalmasso C, Cozzone PJ, Bendahan D. A strictly noninvasive MR setup dedicated to longitudinal studies of mechanical performance, bioenergetics, anatomy, and muscle recruitment in contracting mouse skeletal muscle. Magn Reson Med 2010;64(1):262-270.

26. Arnold DL, Bore PJ, Radda GK, Styles P, Taylor DJ. Excessive intracellular acidosis of skeletal muscle on exercise in a patient with a postviral exhaustion/fatigue syndrome. A ${ }^{31} \mathrm{P}$ nuclear magnetic resonance study. Lancet 1984;1(8391):1367-1369.

27. Vanhamme L, van den Boogaart A, Van Huffel S. Improved method for accurate and efficient quantification of MRS data with use of prior knowledge. J Magn Reson 1997;129(1):35-43.

28. Kemp GJ, Ahmad RE, Nicolay K, Prompers IJ. Quantification of skeletal muscle mitochondrial function by ${ }^{31} \mathrm{P}$ magnetic resonance spectroscopy techniques: a quantitative review. Acta Physiol (Oxf) 2015;213(1):107-144.

29. Kemp GJ, Radda GK. Quantitative interpretation of bioenergetic data from ${ }^{31} \mathrm{P}$ and ${ }^{1} \mathrm{H}$ magnetic resonance spectroscopic studies of skeletal muscle: an analytical review. Magn Reson Q 1994;10(1):43-63.

30. Farvid MS, Ng TW, Chan DC, Barrett PH, Watts GF. Association of adiponectin and resistin with adipose tissue compartments, insulin resistance and dyslipidaemia. Diabetes Obes Metab 2005;7(4):406-413.

31. Arch JR, Hislop D, Wang SJ, Speakman JR. Some mathematical and technical issues in the measurement and interpretation of open-circuit indirect calorimetry in small animals. Int J Obes (Lond) 2006;30(9): 1322-1331.

32. Tschop MH, Speakman JR, Arch JR, Auwerx J, Bruning JC, Chan L, et al. A guide to analysis of mouse energy metabolism. Nat Methods 2011;9(1):57-63

33. Lee SJ, Reed LA, Davies MV, Girgenrath S, Goad ME, Tomkinson KN, et al. Regulation of muscle growth by multiple ligands signaling through activin type II receptors. Proc Natl Acad Sci U S A 2005;102(50): 18117-18122.

34. Cadena SM, Tomkinson KN, Monnell TE, Spaits MS, Kumar R, Underwood KW, et al. Administration of a soluble activin type IIB receptor promotes skeletal muscle growth independent of fiber type. J Appl Physiol 2010;109(3):635-642.

35. Baligand C, Gilson H, Menard JC, Schakman O, Wary C, Thissen JP, et al. Functional assessment of skeletal muscle in intact mice lacking myostatin by concurrent NMR imaging and spectroscopy. Gene Ther 2009;17(3):328-337.

36. Ploquin C, Chabi B, Fouret G, Vernus B, Feillet-Coudray C, Coudray C, et al. Lack of myostatin alters intermyofibrillar mitochondria activity, unbalances redox status, and impairs tolerance to chronic repetitive contractions in muscle. Am J Physiol Endocrinol Metab 2012;302(8): E1000-E1008.

37. Choi SJ, Yablonka-Reuveni Z, Kaiyala KJ, Ogimoto K, Schwartz MW, Wisse BE. Increased energy expenditure and leptin sensitivity account for low fat mass in myostatin-deficient mice. Am J Physiol Endocrinol Metab 2011;300(6):E1031-E1037.

38. McPherron AC, Lee SJ. Suppression of body fat accumulation in myostatin-deficient mice. J Clin Invest 2002;109(5):595-601.

39. Hogan MC, Ingham E, Kurdak SS. Contraction duration affects metabolic energy cost and fatigue in skeletal muscle. Am J Physiol 1998;274: E397-E402.

40. Smith SA, Montain SJ, Matott RP, Zientara GP, Jolesz FA, Fielding RA. Effects of creatine supplementation on the energy cost of muscle contraction: a ${ }^{31}$ P-MRS study. J Appl Physiol 1999;87(1):116-123.

41. Giannesini B, Izquierdo M, Le Fur Y, Cozzone PJ, Bendahan D. In vivo. reduction in ATP cost of contraction is not related to fatigue level in stimulated rat gastrocnemius muscle. J Physiol (Lond) 2001;536(3):905-915.

42. Augusto V, Padovani CR, Rocha Campos GE. Skeletal muscle fiber types in C57B16J mice. Braz J Morphol Sci 2004;27:89-94.

43. Crow MT, Kushmerick MJ. Chemical energetics of slow- and fast-twitch muscles of the mouse. J Gen Physiol 1982;79(1):147-166. 
44. Sawka MN, Petrofsky JS, Phillips CA. Energy cost of submaximal isometric concentrations in cat fast and slow twitch muscles. Pflugers Arch 1981;390(2):164-168.

45. Clausen $\mathrm{T}$. The $\mathrm{Na}^{+}, \mathrm{K}^{+}$pump in skeletal muscle: quantification, regulation and functional significance. Acta Physiol Scand 1996;156(3):227-235.

46. Allen DG, Westerblad H. Role of phosphate and calcium stores in muscle fatigue. J Physiol 2001;536(Pt 3):657-665.

47. Oddoux S, Brocard J, Schweitzer A, Szentesi P, Giannesini B, Brocard J, et al. Triadin deletion induces impaired skeletal muscle function. J Biol Chem 2009;284(50):34918-34929.
48. Amthor H, Macharia R, Navarrete R, Schuelke M, Brown SC, Otto A, et al. Lack of myostatin results in excessive muscle growth but impaired force generation. Proc Natl Acad Sci U S A 2007;104(6): 1835-1840.

49. Bodnar D, Geyer N, Ruzsnavszky O, Olah T, Hegyi B, Sztretye M, et al. Hypermuscular mice with mutation in the myostatin gene display altered calcium signalling. J Physiol 2014;592(Pt 6):1353-1365.

50. Bergstrom M, Hultman E. Energy cost and fatigue during intermitten electrical stimulation of human skeletal muscle. J Appl Physiol 1988; 65(4):1500-1505 